\title{
Paeoniflorin Attenuates Inflammatory Pain by Inhibiting Microglial Activation and Akt-NF-kB Signaling in the Central Nervous System
}

\author{
Bo Hu ${ }^{a}$ Guangtao Xu Xiaomin Zhang ${ }^{\mathrm{b}}$ Long Xua Hong Zhou ${ }^{\mathrm{a}}$ \\ Zhenyi Ma ${ }^{\mathrm{a}}$ Xiaohua Shen ${ }^{\mathrm{a}}$ Jia Zhu ${ }^{\mathrm{a}}$ Ruilin Shen ${ }^{\mathrm{a}}$ \\ aDepartment of Pathology, Institute of Diabetes and Nerve Disease, Jiaxing Hospital of Traditional \\ Chinese Medicine, Jiaxing University, Jiaxing, ZJ, bDepartment of Physiology, Kunming Medical \\ University, Kunming, YN, PR China
}

\section{Key Words}

Paeoniflorin • Freund's complete adjuvant • Pain • Microglia • Akt • NF-kB

\begin{abstract}
Background/Aims: Paeoniflorin (PF) is known to have anti-inflammatory and paregoric effects, but the mechanism underlying its analgesic effect remains unclear. The aim of this study was to clarify the effect of PF on Freund's complete adjuvant (CFA)-induced inflammatory pain and explore the underlying molecular mechanism. Methods: An inflammatory pain model was established by intraplantar injection of CFA in C57BL/6J mice. After intrathecal injection of PF daily for 8 consecutive days, thermal and mechanical withdrawal thresholds, the levels of inflammatory factors TNF- $\alpha$, IL-1 $1 \beta$ and IL- 6 , microglial activity, and the expression of AktNF- $k B$ signaling pathway in the spinal cord tissue were detected by animal ethological test, cell culture, enzyme-linked immunosorbent assay, immunofluorescence histochemistry, and western blot. Results: PF inhibited the spinal microglial activation in the CFA-induced pain model. The production of proinflammatory cytokines was decreased in the central nervous system after PF treatment both in vivo and in vitro. PF further displayed a remarkable effect on inhibiting the activation of Akt-NF-KB signaling pathway in vivo and in vitro. Conclusion: These results suggest that PF is a potential therapeutic agent for inflammatory pain and merits further investigation.

(C) 2018 The Author(s)

Published by S. Karger AG, Basel
\end{abstract}

\section{Introduction}

Pain is an unpleasant sensory experience induced by noxious stimuli, characterized by a heightened responsiveness to several stimuli (hyperalgesia or allodynia) [1,2]. Nearly a third of the world population experiences clinical pathological pain [3]. Pain is believed to be 
caused by aberrant neuronal responses along the pain transmission pathway from the dorsal root ganglion (DRG) to the spinal cord, thalamus, and cortex [4]. Accumulating evidence indicates that neuroinflammation may play a vital role in the pain transmission pathway. Recent studies have revealed that microglia, the immune effector cells in the central nervous system (CNS) critically contribute to the pathogenesis of inflammatory pain [5].

Microglia is resident macrophages in the CNS and exerts important functions in maintaining homeostasis in the CNS [6, 7]. Activation of microglia occurs in most pathological processes, which is often accompanied by morphologic change and production of cytotoxic molecules, and upregulation of phagocytosis and immune surface antigens [8-10]. Activation of microglia is also involved in the pain transmission pathway of acute and chronic pains such as neuropathic pain, inflammatory pain and cancerous pain [11-13]. It has been found that the activated spinal microglia release bioactive molecules including interleukin-1 (IL-1), tumor necrosis factor- $\alpha$ (TNF- $\alpha$ ), nitric oxide (NO), and brain-derived neurotrophic factor (BDNF), which aggravate the range and duration of pain [14-16].

Chinese herbs are important resources to develop safe and effective candidates for the pain therapy. Paeoniflorin (PF) is a chemical compound as one of the major constituents of an herbal medicine derived from Paeonia lactiflora $[17,18]$. In Paeonia, PF can form new compounds with the addition of phenolic substituents $[19,20]$. PF can improve CNS diseases such as cognitive impairment, depression, and Parkinson's disease [21-25]. The combined use of PF and opioid analgesics could potentiate the analgesic effect of opioid analgesics in the treatment of cancerous pain as compared with the use of opioid analgesics alone [26,27]. Our previous study demonstrated that PF exerted the anti-inflammatory effect by inhibiting the activation of microglia [28].

In the present study, we sought to investigate the effect of PF on microglia in inflammatory pain and discovered that Akt-NF- $\kappa$ B signaling pathway was a critical step in the pathogenesis of inflammatory pain. This finding suggests that PF may prove to be a viable therapeutic candidate for the treatment of inflammatory pain.

\section{Materials and Methods}

\section{Animals}

Male C57BL/6J mice were housed under climate-controlled conditions with a 12-h light/dark cycle (lights on at 8:00 A.M., lights off at 8:00 P.M.) at $25{ }^{\circ} \mathrm{C}$ and humidity of $65 \%$ environment during the entire acclimatization for a week with free access to food and water. All experimental procedures were approved by the Ethical Committee of Jiaxing University Medical College (No. JUMC 2017-009) and performed in accordance with the Guide for the Care and Use of Laboratory Animals of the said university.

\section{Establishment and drug treatment of the inflammatory pain model}

PF was purchased from Preferred Biotech (Chengdu, China). The right hind-foot of mice was hypodermically injected with Freund's complete adjuvant (CFA, $20 \mu \mathrm{L}$ ) once to establish an inflammatory pain model. Sham surgery was performed by injecting the same amount of $0.9 \%$ normal saline (NS) to the right hind-foot as a control. After CFA or NS injection, the mice were administered intrathecally $5 \mu \mathrm{L}$ phosphate buffered solution (PBS) as a vehicle control, or $5 \mu \mathrm{L}$ of $0.5,1,2 \mu \mathrm{g}$ PF daily at 10:00 AM from day 1 to day 8 after CFA injection under narcotization with intraperitoneal injection of $4 \%$ chloral hydrate at 7 $\mathrm{mL} / \mathrm{kg}$ body weight. Fifty mice were equally randomized into three groups: Sham group, CFA group, and CFA+PF group $(0.5,1$, and $2 \mu \mathrm{g} P F)$.

\section{Thermal withdrawal latency testing}

Thermal hyperalgesia was measured using ZH-YLS-6BS thermal pain stimulator (Zhenghua Biolog, Anhui, China). Mice were placed in transparent Plexiglass cages. Radiant heat with an appropriate intensity was applied from underneath the platform to the plantar surface of the hind paw until mice showed positive signs of pain. The time when the mouse began licking or withdrawing its paw was recorded and defined as the hot-plate paw withdrawal latency (hPWL). Each measurement was repeated three times at 15- 


\section{Cellular Physiology Cell Physiol Biochem 2018;47:842-850 \begin{tabular}{c|c|c|} 
DOI: 10.1159/000490076 & $\begin{array}{l}\text { O } 2018 \text { The Author(s). Published by S. Karger AG, Basel } \\
\text { www.karger.com/cpb }\end{array}$
\end{tabular}}

Hu et al.: PF Inhibits Inflammatory Pain by Microglia and Akt-NF- $\kappa B$

min intervals and the mean force evoking reliable withdrawals were taken as the threshold. Tests were performed 1 day before modeling and 1, 2, 3, 7 and 8 days after CFA-injection.

\section{Mechanical withdrawal threshold testing}

Mechanical allodynia was examined by assessing paw withdrawal threshold (PWT) using BIO-EVF3 Electric von Frey (IITC, USA). Mice were placed in transparent Plexiglass cages on the top of an elevated metal mesh floor, and a detector was applied at the central region of the plantar surface of the right hind paw to identify the threshold of pain response directly. Each measurement was repeated three times at 10-min intervals and the mean force evoking reliable withdrawals were taken as the threshold.

\section{Enzyme-linked immunosorbent assay (ELISA)}

Ultimately, animals were anesthetized and perfused transcardially with $4 \%$ paraformaldehyde/PBS (pH 7.4) and sacrificed by decapitation. The spinal cord was immediately dissected, and the contaminating blood was washed away with PBS. The spinal cord tissues (lumbar enlargement segments, ca. L4-5) were homogenized in ice-cold PBS and centrifuged at $3600 \times g$ for $15 \mathrm{~min}$ at $4{ }^{\circ} \mathrm{C}$, and the supernatant was used for the double antibody sandwich ELISA and Western blotting. The contents of inflammatory factors TNF- $\alpha$, IL$1 \beta$, and IL- 6 were measured by ELISA kits (Roche, Germany) according to the manufacturer's instructions.

\section{Immunofluorescence histochemistry}

The L4-5 spinal cord was fixed in the $4 \%$ paraformaldehyde at $4^{\circ} \mathrm{C}$ for $5 \mathrm{~h}$ and then transferred to $30 \%$ sucrose/PBS for $24 \mathrm{~h}$. Floating transverse sections of $10 \mu \mathrm{m}$ were used for immunofluorescence histochemistry. The sections for immunofluorescent staining were blocked in a solution containing $5 \%$ normal goat serum and $0.1 \%$ Triton X-100 for $3 \mathrm{~h}$ at room temperature. Then, the sections were incubated $24 \mathrm{~h}$ at $4^{\circ} \mathrm{C}$ with primary antibodies, Iba1 (rabbit polyclonal anti-Iba1, 1:2000; Wako Pure Chemicals), and Neu-N (mouse monoclonal anti-Neu-N, 1:1000; Sigma). After washing, the sections were then incubated with the fluorescent-conjugated secondary antibody (Alexa 488 and Alexa 543, 1:1000; Invitrogen, CA) for $2 \mathrm{~h}$ at room temperature. Fluorescent images were obtained with a confocal microscope (Olympus, Japan). Image J software (NIH, Bethesda, MD) was used to analyze cell images.

\section{Cell culture}

The murine BV2 cell line was maintained in Dulbecco's minimum essential medium (DMEM) supplemented with $10 \%$ fetal bovine serum (FBS), $100 \mathrm{U} / \mathrm{mL}$ penicillin, and $100 \mu \mathrm{g} / \mathrm{mL}$ streptomycin at 37 ${ }^{\circ} \mathrm{C}$ in a humidified incubator with $5 \% \mathrm{CO}_{2}$. The cells were put into a 12 -well plate $\left(5.6 \times 10^{5} / \mathrm{mL}\right)[29]$. BV2 cells cultured were equally randomized into three groups: (1) pure BV2 cell group as control group (CTL group); (2) lipopolysaccharide group (LPS group), where BV2 cells were incubated with LPS ( $0.1 \mu \mathrm{g} / \mathrm{mL}$ ) for $24 \mathrm{~h}$; and (3) LPS+PF group, where cells were co-incubated with LPS $(0.1 \mu \mathrm{g} / \mathrm{mL})$ and PF (200 $\mu \mathrm{M})$ for 24 h. Further, cells were fixed with $4 \%$ preheated neutral paraformaldehyde at $37^{\circ} \mathrm{C}$ for $15 \mathrm{~min}$ and incubated at room temperature for $50 \mathrm{~min}$ with Alexa-Fluor-594 conjunct phalloidine $(50 \mathrm{mg} / \mathrm{mL})$ after treatment with PBS containing $0.1 \%$ Triton X-100. The cell structure was observed under a confocal microscope. The culture media were centrifuged at $3600 \times g$ for $5 \mathrm{~min}$ at $4{ }^{\circ} \mathrm{C}$ to harvest the cell supernatant.

\section{Western blotting}

The western blotting analysis was performed as previously described [30]. Briefly, an equal amount of proteins was loaded and fractionated on $12 \%$ sodium dodecyl sulfate-polyacrylamide gels (SDS-PAGE) and then electrophoretically transferred to the polyvinylidene fluoride (PVDF) membrane (Millipore, Billerica, MA), immunoblotted with antibodies, and visualized by enhanced chemiluminescence (Pierce Biotechnology, Rockford, IL). Film signals were digitally scanned and quantitated using Image J. Densitometric analysis of target protein levels were normalized to an internal control (GAPDH or Lamine B2) and expressed as a relative value.

\section{Statistical analysis}

Data are expressed as mean \pm standard error of mean (SEM). $n$ represents the number of tested animals or cells. The one-way analysis of variance (ANOVA) was used for data analysis. Difference associated with $P<0.05$ was considered statistically significant. 
Results
PF attenuates tactile
allodynia after CFA-
injection

There was no significant difference in hPWL and PWT between the three groups before CFA injection. From postoperative day 1 , the frequency of hind paw withdrawals elicited by mechanical and thermal stimulus was significantly higher in CFA group than that in Sham group $(P<0.05)$ (Fig. 1). In contrast, repeated intrathecal administrations of PF markedly suppressed this decrease in hPWL and PWT in a dose-dependent manner $(P<0.05)$ (Fig. $1)$. These data indicate that $P F$ prevented the development of tactile allodynia after CFA injection.

PF reduces proinflammatory cytokine expression after CFA injection

Knowing that proinflammatory cytokine expression is increased in the process of inflammatory pain [31], we detected TNF- $\alpha$, IL- 6 , and IL- $1 \beta$ in the spinal cord to see whether PF could relieve pain by inhibiting the production of proinflammatory cytokines in the CNS. It was found that the expression of TNF- $\alpha$, IL- 6 , and IL- $1 \beta$ expression induced by CFA was reduced significantly after PF treatment (Fig. 2), indicating that PF could inhibit the expression of inflammatory cytokines in the CNS.

\section{PF inhibits microglial activation after CFA injection}

Knowing that microglia activation and subsequent proinflammatory cytokine expression are responsible for enhanced pain hypersensitivity [32], we used Iba-1 as the microglial activation marker to see whether PF played a role in the activation of spinal microglia in the CFA-induced inflammatory pain mice. It was found that the fluorescence intensity in the spinal cord dorsal horn of CFA-injected mice was increased by more than $50 \%$ compared with that in Sham group, and the expression of Iba-1 was reduced after PF treatment (Fig. $3 \mathrm{~A}$ ). In addition, we also tested another microglial activation marker CD11b and found that the expression of the CD11b protein was up-regulated by 9-fold in CFA and CFA+PF groups (Fig. 3B). There was no significant change in neurons in the three groups. These results suggest that PF could relieve pain by inhibiting the activation of microglia in the CNS in the CFA-induced pain model. 


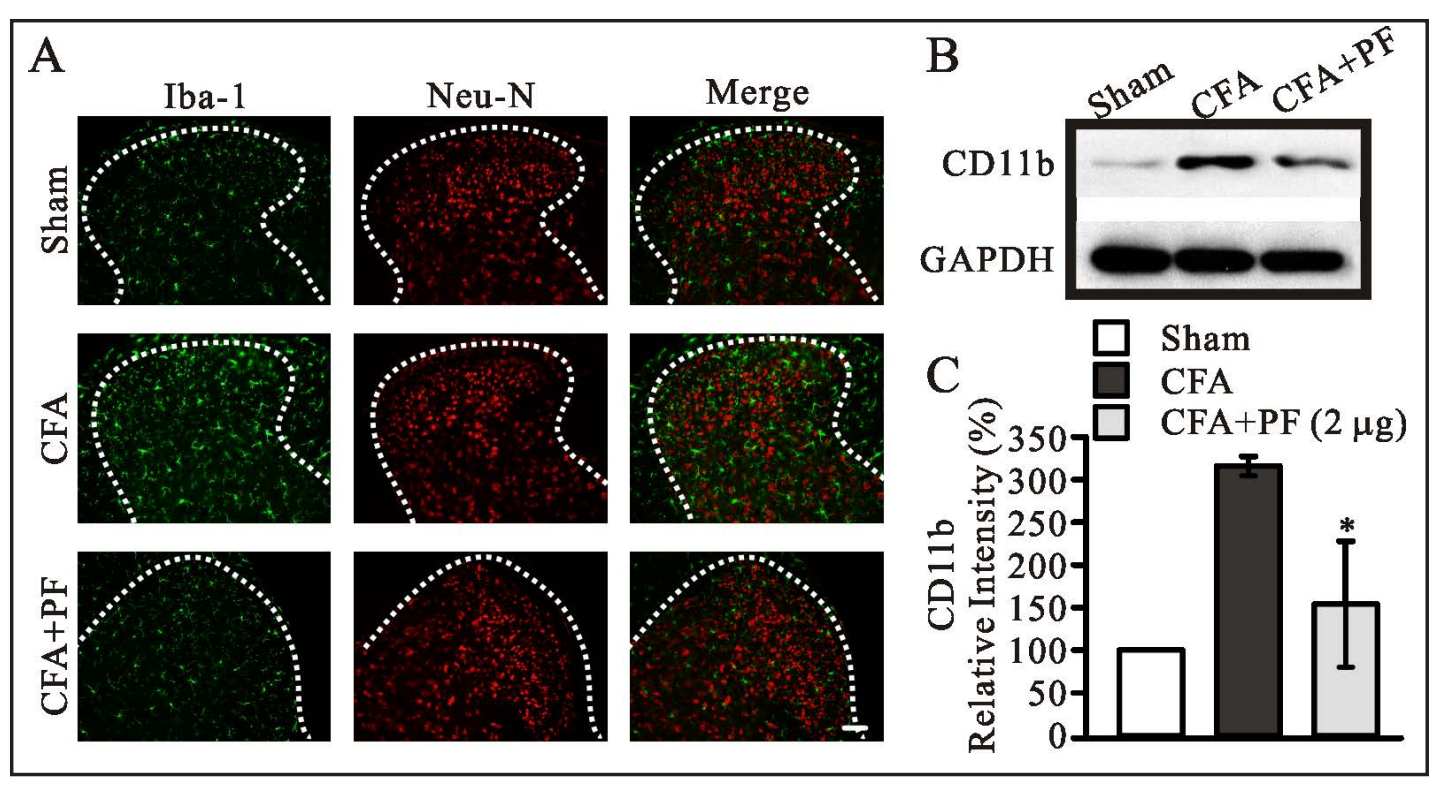

Fig. 3. The effect of PF on microglia activation in the spinal cord. (A) Spinal microglia and neuron were observed by immunohistochemistry using the microglia marker Iba-1 and the neuronal marker Neu-N, respectively. Dotted lines showed the dorsal horn of spinal cord. Scale bar is $200 \mu \mathrm{m}$. (B) The western blotting analysis was used to test the expression of CD11b in the spinal cord. (C) Graphic representation of the relative expression of CD11b normalized to GAPDH. The data represent the means \pm SEM. Note: ${ }^{*} \mathrm{P}<0.05$ vs. CFA.

PF inhibits microglial activation and proinflammatory cytokine expression in vitro

BV2 cells were used to test the effect of PF. It is found that BV2 cells were classified as ramified in the resting state and turned to the hypertrophied type after $24-\mathrm{h} 0.1 \mu \mathrm{g} / \mathrm{mL}$ LPS treatment (Fig. 4A). Knowing that microglia activation was also associated with F-actin rearrangement, we further tested the pattern of F-actin by using phalloidin staining. In the control group, LPS-stimulation caused relatively actin polymerization, as represented by concentrated phalloidin staining in the periphery of the plasma membrane. In contrast, actin aggregation induced by LPS-stimulation was markedly decreased after PF treatment (Fig. 4B).

Knowing that microglia activation produces more proinflammatory cytokine [16], we used ELISA kit to test whether PF could attenuate the release of proinflammatory cytokines, and found that the expression of TNF- $\alpha$, IL- 6 , and IL-1 $\beta$ in the culture medium was increased after LPS-treatment, and reduced after PF intervention (Fig. 4C). Taken together, these data indicate PF could inhibit LPS-induced microglial activation and proinflammatory cytokine production.

Akt-NF- $\kappa B$ signal pathway may be involved in the analgesic mechanism of PF

Knowing that Akt-NF- $\kappa B$ pathway worked as a modulator in many pain models [33, 34], we detected the expression of phosphorylated Akt (p-Akt) in the spinal cord under the same experimental conditions and found that its expression was significantly increased in CFA-induced pain modeled mice, while PF treatment inhibited the increase of p-Akt (p-Akt relative intensity of CFA was $150.38 \pm 2.36 \%$, and CFA+PF was $118.91 \pm 7.41 \%$, Figs. 5A, $\mathrm{C}$, D). Likewise, PF exhibited the similar effect in vitro (p-Akt relative intensity of CFA was $180.31 \pm 20.60 \%$, and CFA+PF was $142.27 \pm 12.99 \%$, Figs. 5 A, F ).

The expression of phosphorylated NF- $\mathrm{BB}-\mathrm{p} 65$ and its nuclear translocation were increased during pain [35]. After CFA injection, the expression of NF- $\kappa B-p 65$ in the spinal cord was increased significantly compared with that in Sham group mice (NF- $\kappa$ B-p65 relative intensity was $234.25 \pm 20.03 \%$ in CFA group, and $127.25 \pm 23.22 \%$ in CFA+PF group, Figs. 5A,

\section{KARGER}


D ). Meanwhile, the expression of NF- $\mathrm{BB}-\mathrm{p} 65$ in the nucleus was also increased in CFA-injected mice(the relative expression of NF- $\mathrm{B}-\mathrm{p} 65$ in CFA group was $186.05 \pm 36.03 \%$, Figs. 5B, F). But the expression and distribution of NF- $\kappa B-p 65$ returned to normal after PF treatment (the NF- $\kappa B-p 65$ relative intensity was $126.86 \pm 40.77 \%$ in $\mathrm{CFA}+\mathrm{PF}$ group, Figs. 5A, B, F). Likewise, PF exhibited the similar effect in vitro (Figs. 5A, B, D, F).

Knowing that $\mathrm{I} \kappa \mathrm{B} \alpha$ binds to NF- $\kappa B$ molecule in cytoplasm to inhibit the activation and nuclear translocation of NF- $\mathrm{BB}$ molecule and the production of NF-кB-p65 molecule is usually accompanied by the degradation of I $\mathrm{B} \alpha \alpha$ molecule [36, 37], we detected the expression of IкB $\alpha$ molecule in the spinal cord and found that I $\mathrm{B} \alpha$ expression was decreased in CFA-injected mice. Compared with Sham group, the expression of $\mathrm{I} \kappa \mathrm{B} \alpha$ was significantly increased after PF intervention. We also found the similar results in BV2 cells in vitro (the relative expression of I $\mathrm{KB} \alpha$ was $29.54 \pm 17.63 \%$ in CFA group, and $105.18 \pm 27.99 \%$ in $\mathrm{CFA}+\mathrm{PF}$ group, Figs. 5A, E). These results suggest that the Akt-NF- $\mathrm{KB}$ signal pathway might be involved in the

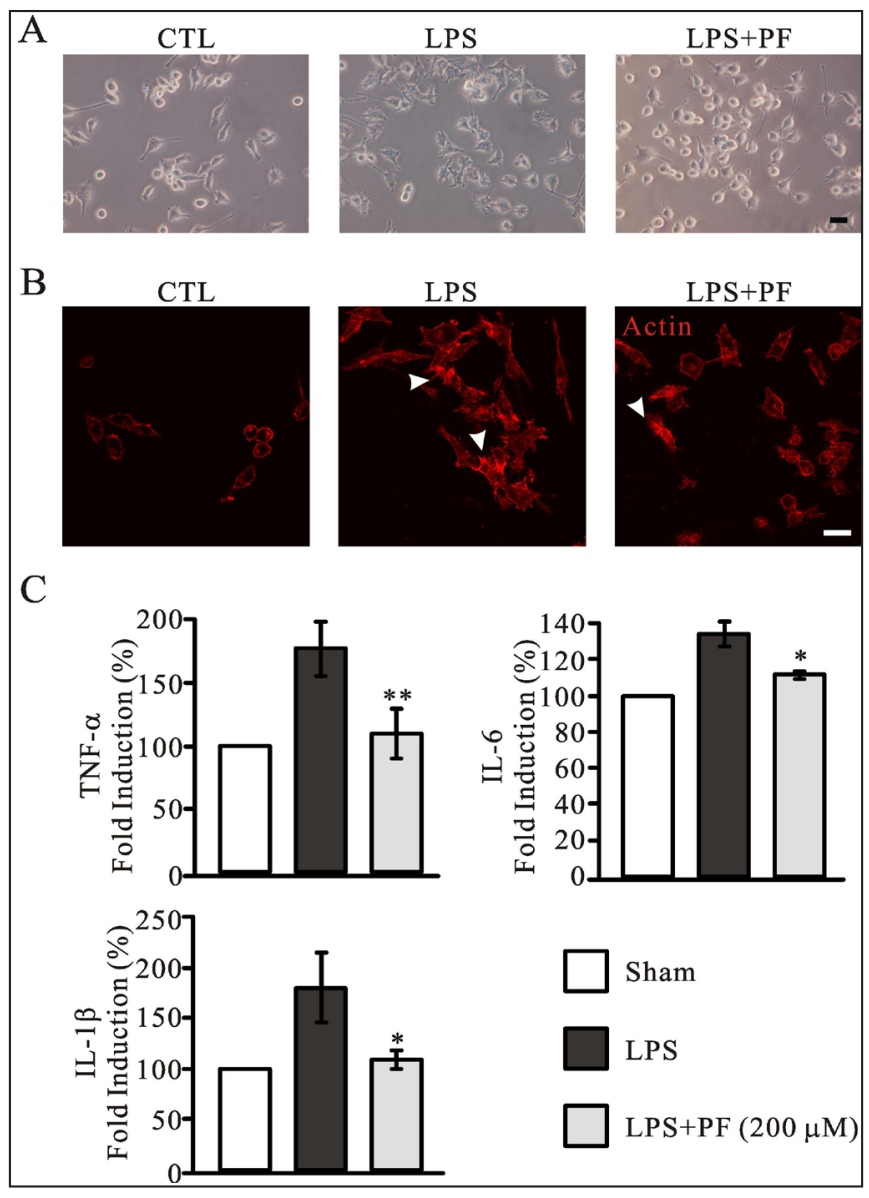

Fig. 4. The effect of PF on microglia activation in vitro. (A) BV2 cells were observed under the bright filed to see their morphological changes after $200 \mu \mathrm{M}$ PF treatment. (B) PF inhibited LPSinduced actin polymerization. Cells were stained with phalloidin (red). F-actin aggregation was in the periphery of BV2 cells (white arrows). Scale bars: $20 \mu \mathrm{m}$. (C) PF inhibited the expression of proinflammatory cytokine TNF- $\alpha$, IL- 6 , and IL-1 $\beta$ in vitro. Note: ${ }^{\#} \mathrm{P}<0.05$ vs. Sham; ${ }^{*} \mathrm{P}<0.05$ vs. LPS. analgesic effect of PF.

\section{Discussion}

Inflammatory pain is the most common type of pain mostly caused by trauma and microbial infection [36]. Nonsteroidal anti-inflammatory drugs (NSAIDs) are commonly used for analgesic treatment in clinical practice. But as NSAIDs often cause severe gastrointestinal adverse effects, it is necessary to find new analgesic drugs with minimal adverse effects. The research showed that PF had good anti-inflammatory and analgesic effects, especially when it was used in combination with morphine $[26,27]$. Nevertheless, the analgesic mechanism of PF is poorly understood yet. In this study, we found that the release of inflammatory factors was reduced after intrathecal injection of PF in the CFA-induced pain mouse model, and PF-treatment could inhibit microglia activation in the CNS.

Microglia is resident macrophages in the CNS. In the model of CFA-induced inflammatory pain, a marked pain threshold was observed on the first day after CFA injection. PF-treatment could significantly increase the threshold of mechanical pain and thermal pain, decrease the 


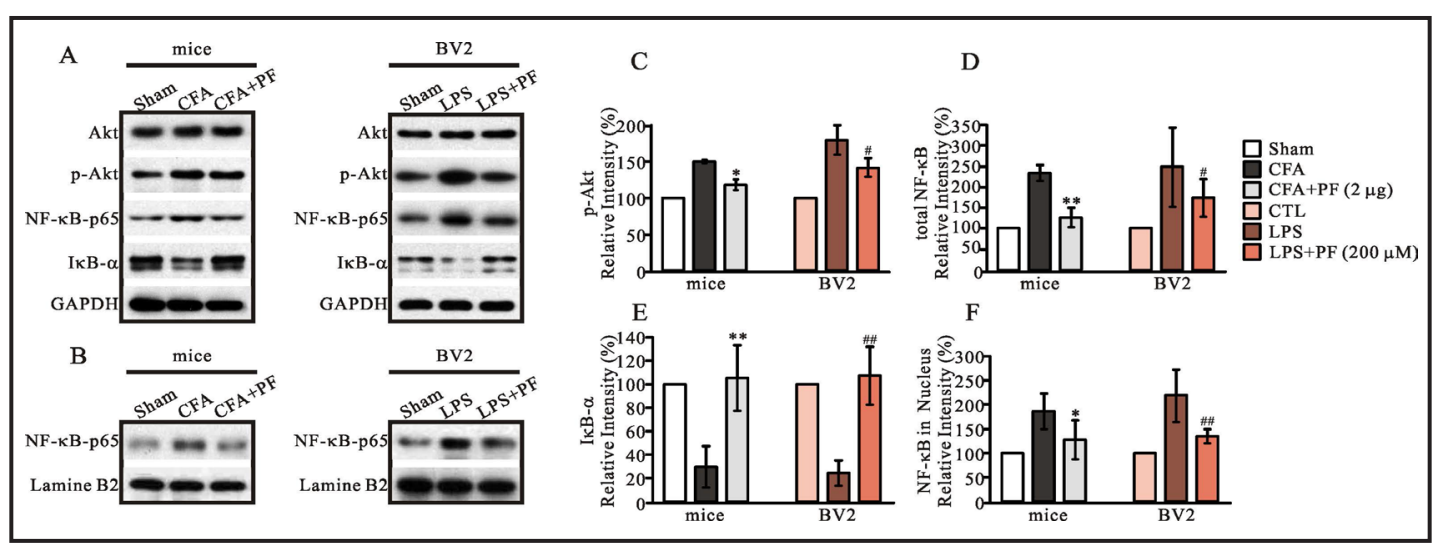

Fig. 5. The Akt-NF- $\kappa B$ signaling pathway is involved in the analgesic effect of PF in vivo/vitro. (A) Western Blotting analysis for the expression of Akt, p-Akt, NF- $\kappa B-p 65$, and I $\kappa \mathrm{B} \alpha$ in the spinal cord and in BV2 cells. (B) Nuclear extracts from each group were tested by Western blotting. Lamine B2 was used as loading

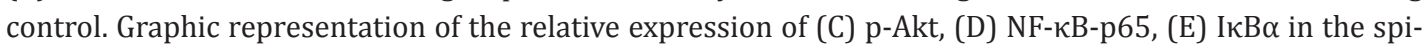
nal cord, and $(\mathrm{F})$ nuclear extracts. The data represent the means $\pm \mathrm{SEM}$. Note: ${ }^{*} \mathrm{P}<0.05,{ }^{* *} \mathrm{P}<0.01$ vs. CFA; ${ }^{\#} \mathrm{P}<0.05$, ${ }^{\# \#} \mathrm{P}<0.01$ vs. LPS.

release of TNF- $\alpha$, IL- 6 , and IL-1 $\beta$, and inhibit microglia activation and Akt-NF- $\kappa B$ expression. PF attenuated microglial activation and TNF- $\alpha$, IL-6, IL-1 $\beta$ production directly in vitro. These results indicate that PF plays an analgesic role by inhibiting microglia in the CNS.

Knowing that inflammation is the main contributing factor of inflammatory pain, we observed that analgesic effect of PF and explored its inhibitory mechanism. It was found in previous studies that PF could inhibit the inflammatory response of dendritic cells by blocking the signal pathway of toll-like receptor $4 / 5$ (TLR4/5) [37], and PF inhibited the synthesis of TNF- $\alpha$, NO, prostaglandin E2 (PE2) and other proinflammatory factors in the mononuclear macrophage system to relieve inflammation [38]. PF also had an inhibitory effect on inflammation in the CNS. It was found that A $\beta 1-42$-induced microglia inflammation could be effectively inhibited by PF. In vivo experiments also confirmed that the release of TNF- $\alpha$ and NO in the midbrain of the mouse Alzheimer's model was inhibited, and the memory and learning function of the mice were improved [39]. We found that PF could inhibit the activation of microglia, and cytokine synthesis, in vivo and in vitro, suggesting that the anti-inflammatory effect of PF is an important mechanism of analgesia.

In summary, PF could inhibit the activation of microglia through the Akt-NF- $\kappa B$ signaling pathway, and therefore may prove to be a potential therapeutic drug for the treatment of inflammatory pain. Further detailed studies are needed to better clarify the underlying mechanism.

\section{Acknowledgements}

The authors greatly appreciate the editors and two anonymous peer reviewers for their critical reading and insightful comments, which have improved our manuscript substantially. This work was supported by grants from the National Natural Science Foundation of China (81501043, 31460258); the Science and Technology Planning Project (Laboratory Animal Project) of Zhejiang Province (2015C37130); and the Science and Technology Planning Project of Jiaxing (2017AY33056, 2018AY35002). The sponsors of the study had no role in study design, data collection, data analysis, data interpretation, or manuscript writing.

\section{Disclosure Statement}

The authors report no conflict of interests in this work. 


\section{Cellular Physiology Cell Physiol Biochem 2018;47:842-850

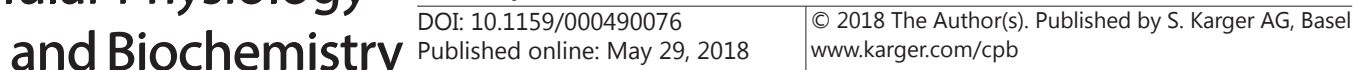

Hu et al.: PF Inhibits Inflammatory Pain by Microglia and Akt-NF-KB

\section{References}

1 Braz JM, Sharif-Naeini R, Vogt D, Kriegstein A, Alvarez-Buylla A, Rubenstein JL, Basbaum AI: Forebrain gabaergic neuron precursors integrate into adult spinal cord and reduce injury-induced neuropathic pain. Neuron 2012;74:663-675.

- Gu ZH, Wang B, Kou ZZ, Bai Y, Chen T, Dong YL, Li H, Li YQ: Endomorphins: Promising endogenous opioid peptides for the development of novel analgesics. Neurosignals 2017;25:98-116.

3 Chen B, Li L, Donovan C, Gao Y, Ali G, Jiang Y, Xu T, Shan G, Sun W: Prevalence and characteristics of chronic body pain in china: A national study. Springerplus 2016;5:938.

-4 Wu LJ, Ko SW, Zhuo M: Kainate receptors and pain: From dorsal root ganglion to the anterior cingulate cortex. Curr Pharm Des 2007;13:1597-1605.

-5 Zhao P, Waxman SG, Hains BC: Extracellular signal-regulated kinase-regulated microglia-neuron signaling by prostaglandin e2 contributes to pain after spinal cord injury. J Neurosci 2007;27:2357-2368.

6 Mei XP, Zhou Y, Wang W, Tang J, Wang W, Zhang H, Xu LX, Li YQ: Ketamine depresses toll-like receptor 3 signaling in spinal microglia in a rat model of neuropathic pain. Neurosignals 2011;19:44-53.

7 Chan FK, Chung SS, Ng IO, Chung SK: The rhoa gtpase-activating protein dlc2 modulates rhoa activity and hyperalgesia to noxious thermal and inflammatory stimuli. Neurosignals 2012;20:112-126.

$>8$ Hanisch UK, Kettenmann H: Microglia: Active sensor and versatile effector cells in the normal and pathologic brain. Nat Neurosci 2007;10:1387-1394.

-9 Aloisi F: Immune function of microglia. Glia 2001;36:165-179.

10 Masaki E, Mizuta K, Ohtani N, Kido K: Early postoperative nociceptive threshold and production of brainderived neurotrophic factor induced by plantar incision are not influenced with minocycline in a rat: Role of spinal microglia. Neurosignals 2016;24:15-24.

11 Peng J, Gu N, Zhou L, U BE, Murugan M, Gan WB, Wu LJ: Microglia and monocytes synergistically promote the transition from acute to chronic pain after nerve injury. Nat Commun 2016;7:12029.

12 Echeverry S, Shi XQ Yang M, Huang H, Wu Y, Lorenzo LE, Perez-Sanchez J, Bonin RP, De Koninck Y, Zhang J: Spinal microglia are required for long-term maintenance of neuropathic pain. Pain 2017;158:1792-1801.

13 Tsuda M, Masuda T, Tozaki-Saitoh H, Inoue K: Microglial regulation of neuropathic pain. J Pharmacol Sci 2013;121:89-94.

-14 Coull JA, Beggs S, Boudreau D, Boivin D, Tsuda M, Inoue K, Gravel C, Salter MW, De Koninck Y: Bdnf from microglia causes the shift in neuronal anion gradient underlying neuropathic pain. Nature 2005;438:10171021.

15 Subedi L, Venkatesan R, Kim SY: Neuroprotective and anti-inflammatory activities of allyl isothiocyanate through attenuation of jnk/nf-kappab/tnf-alpha signaling. Int J Mol Sci 2017;18

-16 Liu Y, Zhou LJ, Wang J, Li D, Ren WJ, Peng J, Wei X, Xu T, Xin WJ, Pang RP, Li YY, Qin ZH, Murugan M, Mattson MP, Wu LJ, Liu XG: Tnf-alpha differentially regulates synaptic plasticity in the hippocampus and spinal cord by microglia-dependent mechanisms after peripheral nerve injury. J Neurosci 2017;37:871-881.

17 Hehir MP, Morrison JJ: Paeoniflorin, a novel heat-shock protein inducing compound, and human myometrial contractility in vitro. J Obstet Gynaecol Res 2016;42:302-306.

-18 Yan D, Saito K, Ohmi Y, Fujie N, Ohtsuka K: Paeoniflorin, a novel heat shock protein-inducing compound. Cell Stress Chaperones 2004;9:378-389.

19 Tanaka T, Kataoka M, Tsuboi N, Kouno I: New monoterpene glycoside esters and phenolic constituents of paeoniae radix, and increase of water solubility of proanthocyanidins in the presence of paeoniflorin. Chem Pharm Bull (Tokyo) 2000;48:201-207.

20 Wu SH, Wu DG, Chen YW: Chemical constituents and bioactivities of plants from the genus paeonia. Chem Biodivers 2010;7:90-104.

21 Zheng M, Liu C, Fan Y, Yan P, Shi D, Zhang Y: Neuroprotection by paeoniflorin in the mptp mouse model of parkinson's disease. Neuropharmacology 2017;116:412-420.

22 Xiao L, Wang YZ, Liu J, Luo XT, Ye Y, Zhu XZ: Effects of paeoniflorin on the cerebral infarction, behavioral and cognitive impairments at the chronic stage of transient middle cerebral artery occlusion in rats. Life Sci 2005;78:413-420.

23 Gu X, Cai Z, Cai M, Liu K, Liu D, Zhang Q, Tan J, Ma Q: Protective effect of paeoniflorin on inflammation and apoptosis in the cerebral cortex of a transgenic mouse model of alzheimer's disease. Mol Med Rep 2016;13:2247-2252. 


\section{Cellular Physiology Cell Physiol Biochem 2018;47:842-850 \begin{tabular}{l|l} 
DOI: 10.1159/000490076 & $\begin{array}{l}\text { O } 2018 \text { The Author(s). Published by S. Karger AG, Basel } \\
\text { www.karger.com/cpb }\end{array}$
\end{tabular}}

Hu et al.: PF Inhibits Inflammatory Pain by Microglia and Akt-NF-kB

24 Yin D, Liu YY, Wang TX, Hu ZZ, Qu WM, Chen JF, Cheng NN, Huang ZL: Paeoniflorin exerts analgesic and hypnotic effects via adenosine a1 receptors in a mouse neuropathic pain model. Psychopharmacology (Berl) 2016;233:281-293.

25 Zhou J, Wang L, Wang J, Wang C, Yang Z, Wang C, Zhu Y, Zhang J: Paeoniflorin and albiflorin attenuate neuropathic pain via mapk pathway in chronic constriction injury rats. Evid Based Complement Alternat Med 2016;2016:8082753.

-26 Chen YF, Lee MM, Fang HL, Yang JG, Chen YC, Tsai HY: Paeoniflorin inhibits excitatory amino acid agonistand high-dose morphine-induced nociceptive behavior in mice via modulation of n-methyl-d-aspartate receptors. BMC Complement Altern Med 2016;16:240.

-27 Jiang C, Xu L, Chen L, Han Y, Tang J, Yang Y, Zhang G, Liu W: Selective suppression of microglial activation by paeoniflorin attenuates morphine tolerance. Eur J Pain 2015;19:908-919.

28 Xu JH, Ao H, Hong JQ Zhou JY, Weng YJ, Zhang JD, Zhu J: Paeoniflorin inhibits lipopolysaccharide-induced microglia inflammation and phagocytosis through il-10-stat3 signaling pathway. Chin J Biochem Mol Biol 2017;33:169-175.

29 Zhu J, Wang Z, Zhang N, Ma J, Xu SL, Wang Y, Shen Y, Li YH: Protein interacting c-kinase 1 modulates surface expression of p2y6 purinoreceptor, actin polymerization and phagocytosis in microglia. Neurochem Res 2016;41:795-803.

-30 Hu B, Wu Y, Liu J, Shen X, Tong F, Xu G, Shen R: Gsk-3beta inhibitor induces expression of nrf2/trxr2 signaling pathway to protect against renal ischemia/reperfusion injury in diabetic rats. Kidney Blood Press Res 2016:937--946.

31 Zhu MD, Zhao LX, Wang XT, Gao YJ, Zhang ZJ: Ligustilide inhibits microglia-mediated proinflammatory cytokines production and inflammatory pain. Brain Res Bull 2014;109:54-60.

-32 Hernangomez M, Klusakova I, Joukal M, Hradilova-Svizenska I, Guaza C, Dubovy P: Cd200r1 agonist attenuates glial activation, inflammatory reactions, and hypersensitivity immediately after its intrathecal application in a rat neuropathic pain model. J Neuroinflammation 2016;13:43.

33 Chen YL, Monteith N, Law PY, Loh HH: Dynamic association of p300 with the promoter of the g proteincoupled rat delta opioid receptor gene during ngf-induced neuronal differentiation. Biochem Biophys Res Commun 2010;396:294-298.

34 Chen YL, Law PY, Loh HH: Action of nf-kappab on the delta opioid receptor gene promoter. Biochem Biophys Res Commun 2007;352:818-822.

35 Miao GS, Liu ZH, Wei SX, Luo JG, Fu ZJ, Sun T: Lipoxin a4 attenuates radicular pain possibly by inhibiting spinal erk, jnk and nf-kappab/p65 and cytokine signals, but not p38, in a rat model of non-compressive lumbar disc herniation. Neuroscience 2015;300:10-18.

36 Schneiderhan J, Orizondo C: Chronic pain: How to approach these 3 common conditions. J Fam Pract 2017;66:145-157.

-37 Zhou Z, Lin J, Huo R, Huang W, Zhang J, Wang L, Sun Y, Shen B, Li N: Total glucosides of paeony attenuated functional maturation of dendritic cells via blocking tlr4/5 signaling in vivo. Int Immunopharmacol 2012;14:275-282.

-38 Sun Y, Dong Y, Jiang HJ, Cai TT, Chen L, Zhou X, Chen T, Xu Q: Dissection of the role of paeoniflorin in the traditional chinese medicinal formula si-ni-san against contact dermatitis in mice. Life Sci 2009;84:337344.

39 Liu H, Wang J: Effect of paeoniflorin on the aß1-42-induced inflammation and chemotaxis of microglia. Chin J Neuroimmunol Neurol 2017;24:276-289. 